\title{
HELMINTOS Y SIFONÁPTEROS PARÁSITOS DE CINCO ESPECIES DE ROEDORES EN LOCALIDADES DE LA CUENCA ORIENTAL, EN EL CENTRO DE MÉXICO
}

\author{
JoRge FALCÓN-ORDAZ, ${ }^{1 *}$ RoxANa ACOSTA, ${ }^{2}$ Jesús A. FERNÁNDEZ ${ }^{3}$ \& \\ GEORGINA LIRA-GUERRERO ${ }^{4}$ \\ ${ }^{1}$ Universidad Autónoma del Estado de Hidalgo, Centro de Investigaciones Biológicas, Apdo. Postal 1- \\ 69, Pachuca, C.P. 42001, Hidalgo, México.*<jfalcon.ordaz@gmail.com> \\ ${ }^{2}$ Museo de Zoología “Alfonso L. Herrera”, Departamento de Biología Evolutiva, Facultad de Ciencias, \\ UNAM; Apdo. Postal 70-399, 04510 México D. F., México. <roxana_a2003@yahoo.com.mx> \\ ${ }^{3}$ Department of Biological Sciences and Museum of Natural Science, 119 Foster Hall, Louisiana State \\ University, Baton Rouge, Louisiana 70803. < jferna9@tigers.lsu.edu> \\ ${ }^{4}$ Universidad La Salle Pachuca, Belisario Domínguez No. 202, Col. Centro, C. P. 42000, Pachuca, \\ Hidalgo, México. < georginageolay@yahoo.com.mx> \\ *Autor de correspondencia: <jfalcon.ordaz@gmail.com>
}

Falcón-Ordaz, J., R. Acosta, J. A. Fernández \& G. Lira-Guerrero. 2012. Helmintos y sifonápteros parásitos de cinco especies de roedores en localidades de la Cuenca Oriental, en el centro de México. Acta Zoológica Mexicana (n. s.), 28(2): 287-304.

RESUMEN. La Cuenca Oriental se ubica en los estados de Puebla, Tlaxcala y Veracruz, localizada en el Eje Volcánico Transmexicano; hasta la fecha, sólo se han registrado en la localidad dos especies de helmintos (Caballerolecythus ibunami y Lamotheoxyuris ackerti) y tres sifonápteros (Anomiopsyllus perotensis, Stenistomera alpina y Echidnophaga gallinacea). Para el presente trabajo se realizaron dos muestreos, en junio de 2007 y julio de 2008, los cuales incluyeron cinco localidades de la región, colectándose ocho taxa de helmintos y nueve de pulgas, todos provenientes de Liomys irroratus, Dipodomys phillipsii, Peromyscus difficilis, P. maniculatus y Reithrodontomys megalotis. Se registran nuevos hospederos y localidades para México en siete taxa de helmintos y sólo uno para las pulgas.

Palabras clave: Helmintos, Sifonápteros, Cuenca Oriental, Liomys irroratus, Dipodomys phillipsii, Peromyscus difficilis, P. maniculatus, Reithrodontomys megalotis.

Falcón-Ordaz, J., R. Acosta, J. A. Fernández \& G. Lira-Guerrero. 2012. Helminths and Siphonaptera parasites of five species of rodents in localities of the Oriental Basin, Mexico. Acta Zoológica Mexicana (n. s.), 28(2): 287-304.

ABSTRACT. The Oriental Basin is located in part of the states of Puebla, Tlaxcala and Veracruz, inside of the Trans-Mexican Volcanic Axis; so far, only two helminth species have been recorded (Caballerolecythus ibunami and Lamotheoxyuris ackerti) and three siphonapterans (Anomiopsyllus perotensis, Stenistomera alpina and Echidnophaga gallinacea). Two sampling visits to the area were carried out in

Recibido: 11/04/2011; aceptado: 19/01/2012. 
June of 2007 and July of 2008, including five localities in the region; eight helminth and nine flea taxa were collected from Liomys irroratus, Dipodomys phillipsii, Peromyscus difficilis, P. maniculatus and Reithrodontomys megalotis. New hosts and localities records for seven helminths and one taxa for fleas were found.

Key words: Helminths, Siphonapterans, Oriental Basin, Liomys irroratus, Dipodomys phillipsii, Peromyscus difficilis, P. maniculatus, Reithrodontomys megalotis.

\section{INTRODUCCIÓN}

La Cuenca Oriental es una extensión de territorio árido o semi-árido de 958.6 km², que se extiende en porciones de los estados de Puebla, Tlaxcala y Veracruz (Fig. 1), se localiza en el polígono latitud $19^{\circ} 42^{\prime} 00^{\prime \prime}-18^{\circ} 57^{\prime} 00^{\prime \prime} \mathrm{N}$ y longitud $98^{\circ} 02^{\prime} 24^{\prime}$ '$97^{\circ} 09^{\prime} 00^{\prime}$ 'O, y se caracteriza por tener pastizales alcalinos, y matorrales áridos en los valles, y bosques de coníferas en las montañas ubicadas alrededor, dicha zona se encuentra en el Eje Volcánico Transmexicano (CONABIO 2010; Valdez \& Ceballos 1997). Hasta la fecha solo se han realizado tres estudios de endo y ectoparásitos de roedores en la región en estudio, dos de ellos basados en un ejemplar del roedor endémico Neotoma nelsoni Goldman, 1905 proveniente de la localidad de El Frijol

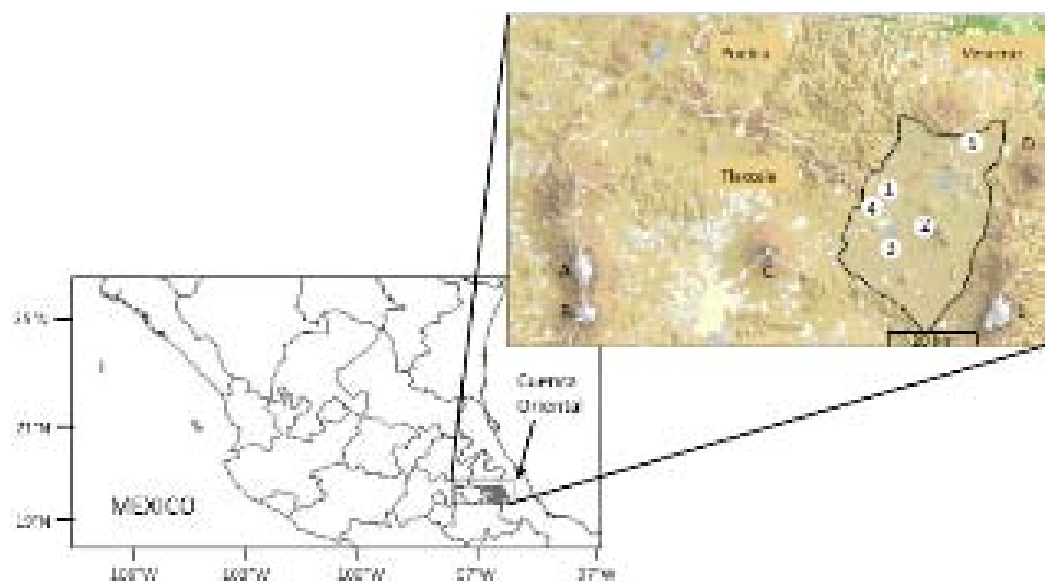

Figura 1. Mapa de la Cuenca Oriental, México (Modificado de http://gaia.inegi. org.mx/mdm5/viewer.html), mostrando los volcanes que le rodean (A = Iztaccihuatl; B = Popocatépetl; C = La Malinche; $\mathrm{D}$ = Cofre de Perote; $\mathrm{E}=$ Pico de Orizaba), y las localidades de colecta: 1 . Puebla: $1.5 \mathrm{~km}$. S Oriental, Municipio de Oriental; 2. Puebla: 2 km. W Guadalupe Victoria, Municipio de Guadalupe Victoria; 3. Puebla: 1 km S Santa Cruz Coyotepec, Municipio de San Salvador El Seco; 4. Tlaxcala: $2.5 \mathrm{Km}$ NO El Carmen Tequexquitla, Municipio de El Carmen Tequexquitla 5. Veracruz: 3 km. S El Frijol Colorado, Municipio de Perote. 
Colorado, Perote, Veracruz, en el cual se registró al nemátodo Lamotheoxyuris ackerti Falcón-Ordaz, et al. 2010, así como a las pulgas Anomiopsyllus perotensis Acosta \& Fernández, 2009, Stenistomera alpina (Baker, 1985) y Echidnophaga gallinacea (Westwood, 1875) (Acosta \& Fernández 2009; Falcón-Ordaz et al. 2010); y en la localidad de El Carmen Tequexquitla, Tlaxcala, se encontró al tremátodo Caballerolecythus ibunami Lamothe-Argumedo et al. 2005 parasitando a dos roedores: Liomys irroratus Gray, 1868 y Peromyscus difficilis Allen, 1891 (Lamothe-Argumedo et al. 2005). Con base en lo anterior, el presente trabajo pretende ampliar el conocimiento de los ecto y endoparásitos presentes en roedores distribuidos en la Cuenca Oriental.

\section{MATERIAL Y MÉTODOS}

Para las colectas de junio de 2007 y julio de 2008 en cinco localidades de la Cuenca Oriental (Fig. 1), se proporcionan a continuación las coordenadas geográficas y altitud (metros sobre el nivel del mar [msnm.]) y la especie de hospedero: México-Puebla: 1.5 km. S Oriental, Municipio de Oriental (19²1'10' 'N, 97³8’07'’O [2365 msnm.]), Liomys irroratus; Peromyscus difficilis; P. maniculatus Wagner, 1845; 2 km. W Guadalupe Victoria, Municipio de Guadalupe Victoria (19¹5’50’’N, 97²2’41'’O [2406 msnm.]), Dipodomys phillipsii Gray, 1841; L. irroratus; P. difficilis; P. maniculatus; Reithrodontomys megalotis Baird, 1857; 1 km S Santa Cruz Coyotepec, Municipio de San Salvador El Seco (1900'33'’N, 97³3'21'’O [2435 msnm.]), L. irroratus; $P$ difficilis; $P$. maniculatus. Tlaxcala: $2.5 \mathrm{Km}$ NO El Carmen Tequexquitla, Municipio de El Carmen Tequexquitla (19²1'0.4'’N, 97³9'55'’O [2378 msnm.]); D. phillipsii; L. irroratus; P. difficilis. Veracruz: $3 \mathrm{~km}$. S El Frijol Colorado, Municipio de Perote (19³4'20'’ N, 97²3'0.7'’O [2435 msnm.]); D. phillipsii; P. difficilis; P. maniculatus; $R$. megalotis.

La captura de roedores fue realizada bajo la autoridad del permiso de colecta FAUT-0002 de la Dirección General de Vida Silvestre de la SEMARNAT otorgado a F. A. Cervantes y se llevó a cabo utilizando métodos estándar aprobados por la American Society of Mammalogists (Gannon et al. 2007; Kelt et al. 2010; Sikes et al. 2011), los ejemplares fueron depositados en la Colección Nacional de Mamíferos (CNMA), del Instituto de Biología de la Universidad Nacional Autónoma de México (U.N.A.M.) y en el Museum of Natural Science, Louisiana State University. Los ejemplares colectados fueron examinados para endo y ectoparásitos; los helmintos se obtuvieron mediante una revisión del tubo digestivo y órganos como hígado, riñones, corazón y pulmones. Los organismos colectados se fijaron y procesaron con base en técnicas convencionales (Lamothe-Argumedo 1997). En el caso de las pulgas, fueron preservadas en alcohol al 70\% y posteriormente montadas siguiendo la metodología de Smit (1957). En la identificación de los organismos de ambos grupos se utilizó literatura especializada, particularmente para los sifonápteros, los trabajos de Traub 
(1950), Hopkins \& Rothschild (1962), Barnes, et al. (1977), Linardi \& Guimarães (2000), Acosta \& Morrone (2003) y el de Hastriter (2004). Los endoparásitos fueron depositados en la Colección Nacional de Helmintos (CNHE), del Instituto de Biología de la U.N.A.M., y los ectoparásitos se encuentran albergados en la Colección de Siphonaptera (MZFC-S) del Museo de Zoología «Alfonso L. Herrera», Facultad de Ciencias, de la U.N.A.M.

\section{RESULTADOS}

Durante la colecta de ecto y endoparásitos se obtuvieron los siguientes taxa: una especie de digéneo, dos de céstodos y cinco de nemátodos, así como nueve especies y subespecies de sifonápteros (Cuadro 1). Enseguida se proporciona la información taxonómica de cada especie colectada, su hospedero, la fecha y localidad de colecta, los números de catálogo de los parásitos colectados y comentarios relevantes a su distribución.

Helmintos

Trematoda Rudolphi, 1808

Dicrocoeliidae Odhner, 1911.

Caballerolecythus ibunami Lamothe-Argumedo, Falcón-Ordaz, García-Prieto \& Fernández, 2005.

Hospedero: L. irroratus.

Prevalencia: 1/6, 16.66\%.

Fecha de colecta: julio-2008.

Localidad: $1.5 \mathrm{Km}$. S Oriental.

Ejemplares depositados: CNHE 7754

Comentarios: El primer registro de esta especie en la zona fue realizado por Lamothe-Argumedo et al. (2005) en L. irroratus y P. difficilis provenientes de El Carmen Tequexquitla, Tlaxcala, localidad que se encuentra en el extremo occidental de la Cuenca Oriental; por lo que en este estudio se realiza el segundo registro de $C$. ibunami en una nueva localidad de la Cuenca.

Cestoidea Rudolphi, 1808

Davaineidae Braun, 1900.

Railletina sp.

Hospedero: D. phillipsii.

Prevalencia: 1/5, 20\% (Puebla); 1/5, 20\% (Veracruz).

Fecha de colecta: junio-2007.

Localidad: $2 \mathrm{Km}$. W Guadalupe Victoria y $3 \mathrm{Km}$. S El Frijol Colorado.

Ejemplares depositados: CNHE 7756, Guadalupe Victoria (Puebla); CNHE 7755, El Frijol Colorado (Veracruz) 


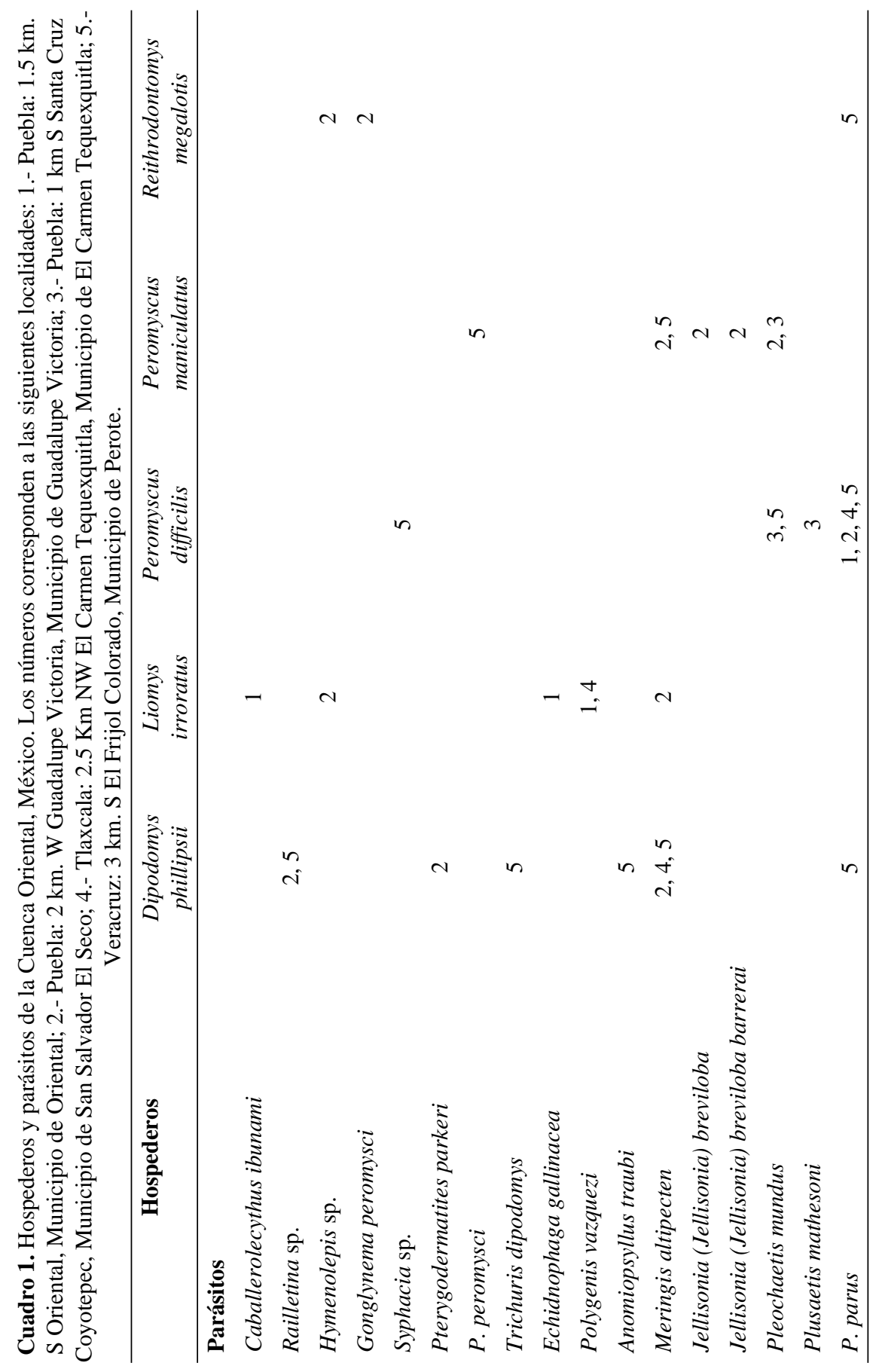


Comentarios: El género Railletina ha sido registrado en diferentes hospederos y localidades de Estados Unidos de América (EUA): Dipodomys merriami Mearns, 1890 y D. ordii Woodhouse, 1853 en Nuevo Mexico (Decker et al. 2001); D. ordii en Texas (Garner et al. 1976); Chaetodipus hispidus (Baird, 1858) y Sigmodon hispidus (Say \& Ord 1825) también en el estado de Texas (Meadows 1968); por lo que esta es la primera vez que se registra en México al género Railletina como parásito de $D$. phillipsii.

Hymenolepididae Railliet \& Henry, 1909.

Hymenolepis sp.

Hospedero: L. irroratus y $R$. megalotis.

Prevalencia: 1/4, 25\% (L. irroratus);1/2, 50\% (R. megalotis).

Fecha de colecta: julio-2008.

Localidad: 2 Km. W Guadalupe Victoria.

Ejemplares depositados: CNHE 7757, L. irroratus; CNHE 7781 R. megalotis.

Comentarios: Es el primer registro en México del género Hymenolepis parasitando a $L$. irroratus y $R$. megalotis, ya que $H$. diminuta ha sido registrada previamente como parásito en $R$. megalotis en el Estado de Washington, EUA (Rankin 1945) y en algunos otros roedores heterómyidos como D. merriami y D. spectabilis Merriam, 1890, en Nuevo Mexico (Decker et al. 2001).

Nematoda Rudolphi, 1808

Gongylonematidae (Hall, 1916 subfam.) Sobolev, 1949

Gongylonema peromysci Kruidenier \& Peebles, 1958.

Hospedero: R. megalotis.

Prevalencia: $1 / 2,50 \%$

Fecha de colecta: julio-2008.

Localidad: $2 \mathrm{Km}$. W Guadalupe Victoria.

Ejemplares depositados: CNHE 7753

Comentarios: Este nemátodo ha sido registrado previamente en $P$. boylii (Baird, 1855), P. eremicus (Baird, 1857), P. maniculatus, P. truei (Shufeldt, 1885) y R. megalotis en Arizona (Kruidenier \& Peebles 1958), el presente registro es el primero en México para la especie.

Oxyuridae Cobbold, 1864

Syphacia (Syphacia) sp.

Hospedero: P. difficilis.

Prevalencia: 1/5, 20\%.

Fecha de colecta: junio-2007.

Localidad: 3 Km. S El Frijol Colorado. 
Ejemplares depositados: CNHE 7752.

Comentarios: En México, el género Syphacia se ha encontrado parasitando a $P$. maniculatus en el estado de Hidalgo (Pulido-Flores et al. 2005; Pulido-Flores 2009), por lo que en este trabajo constituye un nuevo registro de hospedero y localidad para el nemátodo.

Rictularidae (Hall, 1915, subfam.) Railliet, 1916

Pterygodermatites parkeri Lichtenfels, 1970.

Hospedero: D. phillipsii.

Prevalencia: 1/5, 20\%.

Fecha de colecta: julio-2008.

Localidad: $2 \mathrm{Km}$. W Guadalupe Victoria.

Ejemplares depositados: CNHE 7704

Comentarios: Es el primer registro de $P$. parkeri en roedores heterómyidos del género Dipodomys en México, previamente sólo había reportes del helminto en Estados Unidos de América en Sciurus carolinensis Gmelin, 1788 en Virginia; Neotoma magister Baird, 1857 y Napaeozapus insignis (Miller, 1891) en Ohio, Maryland y Connecticut (Lichtenfels 1970).

P. peromysci Lichtenfels, 1970.

Hospedero: P. maniculatus.

Prevalencia: $1 / 2,50 \%$.

Fecha de colecta: julio-2008.

Localidad: $3 \mathrm{Km}$. S El Frijol Colorado.

Ejemplares depositados: CNHE 7751.

Comentarios: P. peromysci se conocía como parásito de diferentes especies de roedores: P. leucopus (Rafinesque, 1818) en el estado de Maryland; P. gossypinus (Le Conte, 1853), P. maniculatus, P. polionotus (Wagner, 1843), Podomys floridanus (Chapman, 1889) y Tamias striatus (Linnaeus, 1758) en los estados de Virginia, Wisconsin, Georgia y Florida (Kinsella 1991 y Lichtenfels 1970) en los Estados Unidos de América, por lo que el presente registro es el primero para México.

Trichuridae (Ransom, 1911) Railliet, 1915

Trichuris dipodomis Read, 1956.

Hospedero: D. phillipsii.

Prevalencia: 1/5, 20\%.

Fecha de colecta: julio-2008.

Localidad: $3 \mathrm{Km}$. S El Frijol Colorado.

Ejemplares depositados: CNHE 7750. 
Comentarios: En el presente estudio se da a conocer un nuevo hospedero para $T$. dipodomis y una nueva localidad. Otros registros del nemátodo han sido en los Estados Unidos de América para D. ordii en Nuevo Mexico y Utah (Grundmann 1957; Read 1956) y para D. microps (Merriam, 1904) en Utah (Grundmann 1957).

Siphonaptera

Pulicidae Billberg, 1820

Echidnophaga gallinacea (Westwood, 1875)

Hospedero: L. irroratus

Prevalencia: $1 / 11,9 \%$

Fecha de colecta: julio-2008

Localidad: $1.5 \mathrm{Km}$. S Oriental.

Ejemplares depositados: 2 hembras, MZFC-S 07454, 07455

Comentarios: Esta especie es considerada ectoparásito primario de aves; sin embargo, existe una lista grande de especies de mamíferos con registros de infestación desde el sur de Estados Unidos hasta Perú, lo que hace suponer que es más común encontrarla asociada a mamíferos que a aves (Hastriter et al. 2002; Bougnton et al. 2006). Esta pulga ha sido ampliamente registrada en México en por lo menos 13 estados (Salceda-Sánchez \& Hastriter 2006), sobre varios huespedes: Canis lupus familiaris Linnaeus, 1758 en el Distrito Federal (D.F.) (Dampf 1925); Otospermophilus variegatus couchi (Baird, 1855) en Nuevo León (Fox 1939; Helgen et al. 2009); Ictidomys mexicanus (Erxleben 1777) en Texcoco, Estado de México; Sylvilagus floridanus orizabae (Merriam, 1893) en Temamatla, Estado de México; Bassariscus astutus Lichtenstein, 1832, Iztapalapa, D.F., Meleagris gallopavo Linnaeus, 1758 en Chapultepec, D. F. (Barrera 1953; Helgen et al. 2009); Mustela f. frenata Lichtenstein, 1831 Huitzilac, Morelos (Barrera 1954a); B. astutus y Didelphis m. mesoamericana J. A. Allen, 1902 (sin embargo, la especie de tlacuache registrada para esa región es D. virginiana californica Bennett, 1833; Hall 1981; Wilson \& Reeder 2005) en Alamos, Sonora (Hubbard 1958); S. floridanus (J. A. Allen, 1890), Lepus callotis Wagler, 1830 y B. astutus en el volcán Popocatépetl (Barrera 1968); Gallus domesticus (Linnaeus, 1758) (Salceda \& Hastriter 2006) en Puebla. Salceda-Sánchez (2004) la reporta para siete estados del país: Guerrero, Jalisco, Michoacán, Puebla, Sinaloa, Tamaulipas y Veracruz; para el estado de Querétaro, Acosta (2003) reporta a esta especie en el hospedero L. irroratus alleni (Coues, 1881) y Peromyscus difficilis saxicola Hoffmeister \& de la Torre, 1959, así como en D. virginiana (Kerr, 1792), L. irroratus y P. levipes Merriam, 1898, Mephitis macroura Lichtenstein, 1832 y B. astutus en Acosta et al. (2008).

Rhopalopsyllidae Oudemans, 1909

Polygenis vazquezi Vargas, 1951. 
Hospedero: L. irroratus.

Prevalencia: 9/11, 81\%

Fecha de colecta: junio-2007 y julio-2008

Localidad: $1 \mathrm{~km}$ S Oriental, $2.5 \mathrm{~km}$ El Carmen Tequexquitla.

Ejemplares depositados: 28 machos, 35 hembras, MZFC-S 07304, 07305, 0731407321, 07401-07418, 07443-07453.

Comentarios: Reportada para siete estados del país (Salceda-Sánchez \& Hastriter 2006). Whitaker et al. (1993) registró a L. pictus (Thomas, 1893) como el único hospedero de la familia Heteromyidae para esta especie de pulga; sin embargo, Acosta et al. (2006) colectaron ejemplares de esta especie en Perognathus flavus Baird, 1865 en el estado de Durango, en donde también la reportaron en otra especie de roedor del género Liomys. También ha sido colectada en Neotomodon alstoni Merriam, 1898 en la carretera México-Cuernavaca; en $P$. boylii (de acuerdo a la taxonomía y distribución aceptadas actualmente, probablemente se trate de $P$. levipes, Wilson \& Reeder 2005), en el estado de Veracruz; L. pictus en Oaxaca, y D. marsupialis Linnaeus, 1758 en Chiapas (Hubbard 1958); N. alstoni en Puebla (Ramírez 1982); Sigmodon hispidus berlandieri Baird, 1855, y L. irroratus alleni en Querétaro (Acosta 2003); Oryzomys chapmani (Thomas, 1898) en Guerrero; S. hispidus y Tadarida brasiliensis (Geoffroy, 1824) en Querétaro; Peromyscus mexicanus (Saussure, 1860) y S. hispidus en Hidalgo y en el género Cryptotis Pomel, 1848 en el estado de San Luis Potosí (Acosta et al. 2008).

Ctenophthalmidae Rothschild, 1915

Anomiopsyllus traubi Barrera, 1951

Hospedero: D. phillipsii

Prevalencia: 1/11, 9\%

Fecha de colecta: junio-2007

Localidad: $3 \mathrm{Km}$. S El Frijol Colorado.

Ejemplares depositados: 2 hembras, MZFC-S 07329, 07330.

Comentarios: Reportada en nido de roedor en el estado de Hidalgo (Barrera 1951; Hopkins \& Rothschild 1962). En este trabajo se presenta el segundo registro para el país.

Meringis altipecten Traub y Hoff, 1951

Hospedero: D. phillipsii, P. maniculatus y L. irroratus.

Prevalencia: 10/11, 90\% (D. phillipsii); 1/5, 20\% (P. maniculatus); 1/11, 9\% (L. irroratus);

Fecha de colecta: junio-2007 y julio-2008.

Localidad: 2 km W Guadalupe Victoria, 2.5 km El Carmen Tequexquitla, 3 km S El Frijol Colorado. 
Ejemplares depositados: 27 machos, 22 hembras, MZFC-S 07328, 07332-07333, 07340-07352, 07354, 07355, 07360, 07397-07400, 07461-07465, 07472-07492.

Comentarios: Reportada ampliamente en el sur de Estados Unidos. Colectada previamente para México en D. merriami, en el estado de Chihuahua (Traub \& Hoff 1951); D. phillipsii perotensis Merriam, 1894 y Peromyscus maniculatus fulvus Osgood, 1904 en Puebla (Barrera 1954b); L. irroratus alleni en Querétaro (Acosta 2003); Onychomys torridus (Coues, 1874) en Durango y L. irroratus en Querétaro (Acosta et al. 2008). Es considerado un parásito habitual del género Dipodomys (Whitaker et al. 1993).

Ceratophyllidae Dampf, 1908

Jellisonia (Jellisonia) breviloba ssp.

Hospedero: P. maniculatus

Prevalencia: 1/5, 20\%

Fecha de colecta: julio-2008.

Localidad: 2 km W Guadalupe Victoria

Ejemplares depositados: 1 hembra, MZFC-S 07493

Comentarios: En México, la especie J. breviloba se ha registrado en 16 estados (Salceda-Sánchez \& Hastriter, 2006). Se ha colectado en P. difficilis felipensis en el Estado de México y en Peromyscus melanophrys (Coues, 1874) en Oaxaca (Acosta et al. 2008). Las condiciones del material colectado no permiten determinarlo hasta nivel subespecífico.

Jellisonia (Jellisonia) breviloba barrerai Hastriter, 2004

Hospedero: P. maniculatus.

Prevalencia: $1 / 5,20 \%$

Fecha de colecta: julio-2008

Localidad: 1 km S Coyotepec.

Ejemplares depositados: 1 hembras, MZFC-S 07369

Comentarios: Reportada para los estados de D.F., Morelos y Puebla por SalcedaSánchez \& Hastriter (2006). Se le ha registrado en Peromyscus aztecus (Saussure, 1860) y Megadontomys thomasi (Merriam, 1898) en Guerrero; P. difficilis y P. maniculatus, en el Distrito Federal; P. difficilis y Neotoma mexicana torquata Ward, 1891 en Morelos; P. difficilis en Puebla (Hastriter 2004); Peromyscus winkelmani Carleton, 1977 y P. levipes en Michoacán; P. aztecus en Oaxaca y Peromyscus sp. en Tlaxcala (Acosta et al. 2008).

Pleochaetis mundus (Jordan \& Rothschild, 1922)

Hospedero: P. difficilis y P. maniculatus.

Prevalencia: 3/5, 60\% (P. maniculatus); 1/5, 20\% (P. difficilis) 
Fecha de colecta: junio-2007 y julio-2008

Localidad: 2 km W Guadalupe Victoria, 1 km S Coyotepec, 3 km S El Frijol Colorado.

Ejemplares depositados: 2 machos, 9 hembras, MZFC-S 07338, 07353, 0735607358, 07366, 07494-07495, 07498-07500.

Comentarios: Esta es una de las especies de pulgas que se encuentran ampliamente distribuidas en el país, con reportes en 15 estados (Salceda-Sánchez \& Hastriter 2006) y colectada en varias especies de roedores. Sin embargo, también existen registros en murciélagos para el estado de Querétaro, así como en ratas de campo y $P$. melanotis en el Distrito Federal, nido de R. c. chrysopsys y P. hylocetes en Michoacán (Traub 1950); S. hispidus en Jalisco, P. melanotis en el D. F. (Dampf 1942); Reithrodontomys megalotis saturatus J. A. Allen y Chapman, 1897 en Hidalgo, $P$. difficilis amplus en Tlaxcala (Barrera 1953); M. m. mexicanus y Peromyscus sp. en el D.F. (Machado-A 1960); M. mexicanus, P. difficilis y P. maniculatus en el volcán Popocatépetl (Barrera 1968); Baiomys taylori (Thomas, 1887), B. taylori analogous (Osgood, 1909), Liomys sp., L. irroratus alleni, Neotoma albigula leucodon Merriam, 1894 (actualmente las poblaciones de Querétaro se reconocen como Neotoma leucodon Merriam, 1894), N. goldmani Merriam, 1903, Peromyscus sp., P. boylii, $P$. difficilis, P. d. difficilis (J. A. Allen, 1891), P. d. saxicola, P. gratus Merriam, 1898, P. l. levipes Merriam, 1898, Peromyscus melanophrys zamorae Osgood, 1904, P. pectoralis Osgood, 1904, Sciurus deppei Peters, 1863, Sigmodon sp., Myotis californicus (Audubon \& Bachman 1842) y Sturnira sp. en Querétaro y P. maniculatus en Tlaxcala (Acosta 2003; Acosta et al. 2008).

Plusaetis mathesoni (Traub, 1950)

Hospedero: P. difficilis.

Prevalencia: $1 / 5,20 \%$

Fecha de colecta: junio-2007.

Localidad: $1 \mathrm{~km}$ S Coyotepec.

Ejemplares depositados: 3 hembras, MZFC-S 07367, 07368, 07370.

Comentarios: Registrada en 11 estados del país por Salceda-Sánchez \& Hastriter (2006). Se les ha encontrado en: Reithrodontomys c. chrysopsys Merriam, 1900 y Peromyscus hylocetes Merriam, 1898 en Michoacán (Traub 1950); P. maniculatus en Hidalgo (Barrera 1953); P. hylocetes, Microtus m. mexicanus Saussure, 1861 y Sorex s. saussueri Merriam, 1892 en Morelos (Barrera 1954a); Peromyscus (Megadontomys) thomasi en Guerrero (Barrera 1958); Neotoma mexicana Baird, 1855 en Chiapas (Hubbard 1958); Microtus m. mexicanus, Reithrodontomys sp., y Peromyscus sp., en el Distrito Federal (Machado-A. 1960); M. m. mexicanus, N. alstoni, P. difficilis, P. maniculatus, Peromyscus melanotis J. A. Allen \& Chapman, 1897, $R$. chrysopsys y R. megalotis en el volcán Popocatépetl (Barrera 1968); M. m. 
mexicanus, P. maniculatus, $R$. megalotis y Rattus norvegicus Berkenhout, 1769 en el Distrito Federal (Méndez-L, 1974); $R$. c. chrysopsys y P. hylocetes en Morelos (Pérez-Ortiz, 1976); Peromyscus d. saxicola Hoffmeister \& de la Torre, 1959, P. l. levipes Merriam, 1898 y Reithrodontomys megalotis amoles A. H. Howell, 1914 en Querétaro (Acosta 2003); P. difficilis en el Estado de México, P. difficilis, P. maniculatus, Peromyscus sp., y Reithrodontomys chrysopsis Merriam, 1900 en el D.F., $M$. thomasi, O. chapmani, P. megalops Merriam, 1898, y Peromyscus sp. en Guerrero, $P$. aztecus, $P$. difficilis, $P$. gratus, $P$. mexicanus y Reithrodontomys mexicanus (Saussure, 1860) en Oaxaca; N. mexicana, P. boylii, P. difficilis, P. levipes, Peromyscus sp., $R$. megalotis, y Sigmodon sp. en Querétaro; L. irroratus, $N$. mexicana, P. difficilis, $P$. gratus, Peromyscus sp., Reithrodontomys sp. en Tlaxcala y también registrado en Peromyscus sp. en Veracruz y en Puebla (Acosta \& Fernández 2006; Acosta et al. 2008).

P. parus (Traub, 1950)

Hospedero: D. phillipsii, P. difficilis y R. megalotis.

Prevalencia: 1/11, 9\% (D. phillipsii); 3/5, 60\% (P. difficilis); 1/1, 100\% (R. megalotis)

Fecha de colecta: junio-2007 y julio-2008.

Localidad: 1 km S Oriental, 2 km W Guadalupe Victoria, 2.5 km El Carmen Tequexquitla, $3 \mathrm{~km} \mathrm{~S}$ El Frijol Colorado.

Ejemplares depositados: 17 machos, 16 hembras, MZFC-S 07306-07313, 0732207327, 07331, 07334-07337, 07359, 07361-07365, 07466-07471, 07496.

Comentarios: Reportada en 7 estados por Salceda-Sánchez \& Hastriter (2006). Algunos de los hospederos en los que se ha encontrado son el ratón Peromyscus truei gratus (las poblaciones del Distrito Federal corresponden a P. gratus; Wilson \& Reeder 2005), P. maniculatus labecula en el D.F.; P. difficilis amplus Osgood 1904, $R$. megalotis saturatus en Hidalgo; $P$. difficilis amplus en Tlaxcala (Barrera 1953); $M$. mexicanus, $P$. difficilis, $P$. maniculatus, $R$. chrysopsys, $R$. megalotis, Mustela frenata perotae Hall, 1936 en el volcán Popocatépetl (Barrera 1968); B. taylori analogous, Oryzomys chapmani huastecae Dalquest, 1951, P. d. difficillis, P. l. levipes, R. mexicanus, Reithrodontomys s. sumichrasti (Saussure, 1861) en Querétaro (Acosta 2003); P. gratus en el Parque Nacional Malinche, (Acosta \& Fernández 2006).

\section{DISCUSIÓN}

En el presente trabajo se enlistan 17 taxa de parásitos asociados a cinco especies de roedores pertenecientes a las familias Heteromyidae (D. phillipsii y L. irroratus) y Cricetidae ( $P$. difficilis, $P$. maniculatus y $R$. megalotis). En el caso de los helmintos se muestra que el grupo con mayor abundancia son los nemátodos con cinco especies, seguido de los céstodos con dos y un digéneo. En el caso de los sifonápteros 
se reportan tres familias de las ocho que existen en el país. Para el grupo de los helmintos se registran siete especies con una nueva localidad y un nuevo hospedero, mientras que $C$. ibunami sólo se encuentra en una nueva localidad. En cuanto a las especies de sifonápteros, Anomiopsyllus traubi representa el segundo registro para el país y un nuevo hospedero, mientras que Meringis altipecten se encuentra en tres de las cinco especies de roedores colectadas (2 heterómyidos y un múrido). Antes del presente estudio únicamente se habían registrado dos especies de helmintos y tres sifonápteros en roedores de esta región (Acosta \& Fernández 2009; Falcón-Ordaz et al. 2010; Lamothe-Argumedo et al. 2005), por lo que el presente trabajo amplía el conocimiento de la fauna de helmintos y sifonápteros para esta zona del país, además de proporcionar por primera vez una lista de helmintos que parasitan a $R$. megalotis y D. phillipsii, este último, roedor endémico de las zonas áridas y semiáridas del centro y norte de México.

De las ocho especies de helmintos colectadas, seis (C. ibunami, Railletina sp., Hymenolepis sp., G. peromysci, P. parkeri y P. peromysci) tienen un ciclo de vida heteroxeno, lo que implica la presencia de uno o dos hospederos intermediarios: un caracol y algún insecto (Anderson 2000; Olsen 1962) y en Syphacia (Syphacia) sp. y T. dipodomis, su ciclo de vida es de tipo monoxeno, por lo que hay una autoinfección. Esta diferencia en el número de especies, determinada por el ciclo de vida, nos indica que el tipo de alimentación durante la época muestreada se basa principalmente en insectos, lo que es común en los hospederos pertenecientes a la familia Heteromyidae, comportamiento que ha sido registrado en varias especies de Dipodomys (Bienek \& Klikoff 1974; King \& Babero 1974; Pfaffenberger et al. 1985) y en L. irroratus se documenta por primera vez este comportamiento al encontrarse parasitado por $C$. ibunami.

Varios autores, entre ellos Ford et al. (2004), mencionan que las pulgas presentan varios grados de especificidad, algunas de ellas tienen una o dos especies de hospederos preferidos, mientras que otras son eurixenas (inespecificas), y pueden parasitar hospederos pertenecientes a un género o hasta a una familia, como sería el caso de Meringis altipecten y Polygenis vazquezi que a lo largo de su distribución, se encuentran asociadas casi siempre a especies de la familia Heteromyidae; en el presente trabajo se encontró a $M$. altipecten sobre $D$. phillipsii y $P$. vazquezi en Liomys irroratus.

De acuerdo con la composición de la biota de helmintos y sifonápteros encontrada en este estudio, los registros de estas especies en diferentes trabajos y los hospederos (Barrera 1968; Morrone \& Gutiérrez, 2005; Acosta \& Fernández, 2006; Escalante et al. 2011), es posible inferir que la Cuenca Oriental tiene elementos con una afinidad Neártica, particularmente con las zonas áridas de la provincia del Altiplano Mexicano y del sur de los Estados Unidos de América (Nuevo Mexico y Texas), ya que se encontraron especies que se distribuyen en dicha provincia (Railletina sp., Hymenolepis 
sp. y T. dipodomis) y en especies del mismo género que las reportadas en el presente; sin embargo, también encontramos una especie de pulga que se considera de afinidad Neotropical (Polygenis vazquezi Ponce y Llorente 1996; Gutiérrez-Velázquez \& Acosta 2004).

Finalmente, y a falta de un estudio con cobertura geográfica más amplia dentro de la Cuenca Oriental, que incluya una mayor cantidad de localidades, de diversidad taxonómica y un tamaño de muestra más grande de los hospederos, así como una mayor cantidad de parásitos, con los datos obtenidos tenemos evidencia preliminar de que los helmintos de esta región en particular, pueden presentar dos tipos de asociación con sus huéspedes: la primera, una coevolución por descendencia, en la cual sus ancestros se asociaron entre sí en el pasado y las especies heredaron dicha asociación, tal es el caso de $T$. dipodomis en $D$. microps, $D$. ordii y $D$. phillipsii, que son especies relacionadas filogenéticamente (Hafner et al. 2007); la segunda posible relación que se puede observar, es producto de una hipotética colonización, en la cual una de las especies se originó en otro huésped y subsecuentemente se relacionaron por transferencia o dispersión, básicamente en las especies con un ciclo de vida heteroxeno (Brooks \& McLennan1993).

Agradecimientos. Los autores agradecen a Fernando Cervantes Reza por el préstamo de los permisos de colecta, a Juan Carlos Winfield-Pérez por su ayuda en la colecta de los roedores, a Berenit Mendoza Garfias y Rogelio Rosas Valdez por la tinción e identificación de los céstodos. Los autores agradecen los comentarios de 3 revisores anónimos. Este artículo fue escrito mientras J.F.O. tuvo una estancia posdoctoral en el Laboratorio de Morfología Animal, Centro de Investigaciones Biológicas, Universidad Autónoma del Estado de Hidalgo, con el programa: "Estancias posdoctorales y sabáticas vinculadas al fortalecimiento de la calidad del Posgrado Nacional 2009”, del Consejo Nacional de Ciencia y Tecnología (CONACyT).

\section{LITERATURA CITADA}

Acosta, R. 2003. New records of rodent fleas from Queretaro, Mexico (Siphonaptera). Zootaxa, 369: 1-15.

Acosta, G. R. \& J. A. Fernández. 2006. Pulgas (Insecta: Siphonaptera), Fauna de pulgas asociadas a mamíferos, pp. 157-174. In: Fernández, J. A. \& J. C. López D. (Compiladores). Biodiversidad Parque Nacional Malinche, Tlaxcala, México. Coordinación General de Ecología.

Acosta, R., J. A. Fernández \& J. Falcón-Ordaz. 2006. New records of mammal fleas (Siphonaptera) in northern and central Mexico. Entomological News, 117: 69-72.

Acosta, R., J. A. Fernández, J. Llorente \& M. C. Jiménez. 2008. Catálogo de pulgas (Insecta: Siphonaptera). Serie Catálogos del Museo de Zoología “Alfonso L. Herrera” 1:1-111.

Acosta, R. \& J. A. Fernández. 2009. A new species of Anomiopsyllus Baker, 1904 (Insecta: Siphonaptera), and noteworthy records of fleas from Nelson's Woodrat, Neotoma nelsoni (Rodentia: Cricetidae), in the Oriental Basin, México. Journal of Parasitology, 95: 532-535.

Acosta, R. \& J. J. Morrone. 2003. Clave ilustrada para la identificación de los taxones supraespecíficos de Siphonaptera de México. Acta Zoológica Mexicana, Nueva Serie, 89: 39-53. 
Anderson, R. C. 2000. Nematode Parasites of Vertebrates: Their Development and Transmission. 2nd ed. CAB International, Wallingford, Oxon, U.K. 650 pp.

Barnes, A. M., V. J. Tipton \& J. A. Wildie. 1977. The subfamily Anomiopsyllinae (Hystrichopsyllidae: Siphonaptera). I. A revision of the genus Anomiopsyllus Baker. Great Basin Naturalist, 37: 138-206.

Barrera, A. 1951. Notas sobre sifonápteros II. Descripción de Anomiopsyllus traubi nov. sp. (Siph., Hystrichops). Ciencia (Méx.), 9: 197-200.

Barrera, A. 1953. Sinopsis de los sifonápteros de la Cuenca de México (Ins., Siph.) Anales de la Escuela Nacional de Ciencias Biológicas, 7: 155-245.

Barrera, A. 1954a. Notas sobre sifonápteros VII. Lista de especies colectadas en el Municipio de Huitzilac; Morelos, y descripción de Peromyscopsylla zempoalensis nov. (Siph., Leptops.). Ciencia (Méx.), 14: 87-90.

Barrera, A. 1954b. Notas sobre sifonápteros. VIII. Nuevas localidades de especies conocidas y nuevas para México y diagnosis de Pleochaetis apollinaris aztecus subsp. Nov. Ciencia (Méx.), 14: 137139.

Barrera, A. 1958. Insectos parásitos de mamíferos salvajes de Omiltemi, Guerrero, y descripción de un nuevo sifonáptero: Pleochaetis soberoni nov. sp. Annales de la Escuela Nacional de Ciencias Biológicas, 9: 89-96.

Barrera, A. 1968. Distribución cliseral de los Siphonaptera del volcán Popocatépetl, su interpretación biogeográfica. Anales del Instituto de Biología, UNAM, 39: 35-100.

Bienek, G. K. \& L. G. Klikoff. 1974. Parasitological evidence of arthropods as food for Dipodomys merriami vulcani. The American Midland Naturalist, 91: 251-253.

Boughton, R. K., J. W. Atwell, \& S. J. Schoech. 2006. An Introduced generalist parasite, the sticktight fleas (Echidnophaga gallinacea), and its pathology in the threatened Florida Scrub-Jay (Aphelocoma coerulescens). Journal of Parasitology, 95: 941-948.

Brooks, D. R. \& D. McLennan. 1993. Parascript: Parasites and the language of evolution. Smithsonian Institution Press. Washington, D.C.

CONABIO: Comisión Nacional para el Conocimiento y uso de la Biodiversidad. 2010. Página en red: http://www.conabio.gob.mx/conocimiento/regionalizacion/doctos/rhp_070.html; (consultada: 15 junio, 2010).

Dampf, A. 1925. Ectoparásitos del perro, colectados en los meses de julio a noviembre de 1924, por el servicio de desinfección y desinsectización del Departamento de Salud Pública de México. Boletin del Departamento de Salud Publica, 2: 33-42.

Dampf, A.1942. Nuevos datos sobre la pulga Pleochaetis mundus (Jordan y Rothschild 1922). Revista de la Sociedad Méxicana de Histirotia Natural, 3: 135-148.

Decker, H. D., D. W. Duszynski \& M. J. Patrick. 2001. Biotic and abiotic effects on endoparasites infecting Dipodomys and Perognathus species. Journal of Parasitology, 87: 300-307.

Escalante, T., E. A. Martínez-Salazar, J. Falcón-Ordaz, M. Linaje \& R. Guerrero. 2011. Análisis panbiogeográfico de Vexillata (Nematoda: Ornithostrongylidae) y sus huéspedes (Mammalia: Rodentia). Acta Zoológica Mexicana (n. s.), 27: 25-56.

Falcón-Ordaz, J., J. A. Fernández \& L. García-Prieto. 2010. Lamotheoxyuris ackerti (Kruidenier and Mehra, 1959) n. gen., n. comb. (Nematoda: Heteroxynematidae) parasite of Neotoma spp. Rodentia: Muridae). Revista Chilena de Historia Natural, 83: 259-266.

Ford, P. L., R. A. Fagerlund, D. W. Duszynski, \& P. J. Polechla. 2004. Fleas and lice of mammals in New Mexico. Gen. Tech. Rep. RMRS-GTR-123. Fort Collins, CO: U. S. Department of Agriculture. Rocky Mountain Research Station, 57 pp. 
Fox, I. 1939. New species and records of Siphonaptera from Mexico. Iowa State College Journal Science, 13: 335-339.

Gannon, W. L., R. S. Sikes \& The Animal Care and use Committee of the American Society of Mammalogists. 2007. Guidlines of the American Society of Mammalogists for the use of wild animals in research. Journal of Mammalogy, 88: 809-823.

Garner, H. W., L. W. Richardson \& L. A. Felts. 1976. Alimentary helminths of Dipodomys ordii: effects on the host population. Southwestern Naturalist, 21: 327-334.

Grundmann, A. W. 1957. Nematode parasites of mammals of the Great Salt Lake Desert of Utah. Journal of Parasitology, 43: 105-112.

Gutiérrez-Velázquez, A. L. \& R. Acosta. 2004. Relaciones biogeográficas basadas en la distribución de Siphonaptera (Insecta). Pp. 393-416. In: Luna, I., J. J. Morrone y E. Espinosa (Eds.), Biodiversidad de la Sierra Madre Oriental, Las Prensas de Ciencias, México D. F.

Hafner, J. C., J. E. Light, D. J. Hafner, M. S. Hafner, E. Reddington, D. S. Rogers \& B. R. Riddle. 2007. Basal clades and molecular systematics of heteromyid rodents. Journal of Mammalogy, 88: 1129-1145.

Hall, E. R. 1981. The mammals of North America. $2^{\text {nd }}$ ed. John Wiley \& Sons, Inc., New York 1:1-600 +90 .

Hastriter, W. M. 2004. Revision of the fleas genus Jellisonia Traub, 1944 (Siphonaptera: Ceratophylllidae). Annals of Carnegie Museum, 73: 213-238.

Hastriter, W. M., M. D. Zyzak, R. Fernandez, N. Solorzano, \& M. F. Whiting. 2002. Fleas (Siphonaptera) from Ancash Department, Peru with the description of a new species, Ectinorus alejoi (Rhopalopsyllidae), and the description of the male of Plocopsylla pallas (Rothschild, 1914) (Stephanopcircidae). Annals of Carnegie Museum, 72: 87-106.

Helgen, K. M., F. Russell C., L. E. Helgen \& D. E. Wilson. 2009. Generic revison of the Holarctic ground squirrels Genus Spermophilus. Journal of Mammalogy, 90: 270-305.

Hopkins, G.H. \& M. Rothschild. 1962. An illustrated catalogue of the Rothschild collection of fleas (Siphonaptera) in the British Museum (Natural History) III. Hystrichopsyllidae (Acedestiinae, Anomiopsyllinae, Hystrichopsyllinae, Neopsyllinae, Rhadinopsyllinae and Stenoponiinae). British Museum (N.H.). 560 pp.

Hubbard, C. A. 1958. Mexican Jungle and fleas with three new descriptions. Entomological News, 69: 161-166.

Kelt, D. A., M. S. Hafner, \& the American Society of Mammalogists' ad hoc Committee for guidelines on handling rodents in the field. 2010. Journal of Mammalogy, 91: 1524-1527.

King, S. R. \& B. B. Babero. 1974. Helminths of kangaroo rats (Dipodomys spp.) in Nevada with reports of other worm parasites from these hosts. Proceedings of the Helminthological Society of Washington, 41: 241-248.

Kinsella, J. M. 1991. Comparison of helminths of three species of mice, Podomys floridanus, Peromyscus gossypinus, and Peromyscus polionotus, from southern Florida. Canadian Journal of Zoology, 69: 3078-3083.

Kruidenier, F. J. \& C. R. Peebles. 1958. Gongylonema of rodents: G. neoplasticum (redefinition); G. dipodomysis n. sp. and G. peromysci n. sp. Transactions of the American Microscopical Sosciety, 77: 307-315.

Lamothe-Argumedo, R. 1997. Manual de técnicas para prepara y estudiar los parásitos de animales silvestres. AGT Editor, S. A. México, D. F. 43 pp.

Lamothe-Argumedo, R., J. Falcon-Ordaz, L. Garcia-Prieto \& J. Fernandez-Fernandez. 2005. A new dicrocoeliid (Digenea: Dicrocoeliinae) parasite of rodents from Tlaxcala, Mexico. Journal of Parasitology, 91: 1410-1412. 
Lichtenfels, J. R. 1970. Two new species of Pterygodermatites (Paucipectines) Quentin, 1969 (Nematoda: Rictulariidae) with a key to the species from North American Rodents. Proceedings of the Helminthological Society of Washington, 37: 94-101.

Linardi M. P. \& L. R. Guimarães. 2000. Sifonápteros do Brasil. São Paulo, Museu de Zoologia, 291 pp.

Machado-A. C. E. 1960. Microtus mexicanus mexicanus (De Saussure, 1861) su biología, ectoparácitos y otras formas animales ecológicamente relacionadas. Tesis Profesional, Facultad de Ciencias, UNAM, México, 105 pp.

Meadows, J. 1968. Parasite community structure in three rodents, Sigmodon, Perognathus and Baiomys, in Central Texas. M. A. Thesis, Baylor University.

Méndez-L. J. 1974. Composición específica y fluctuación del número de sifonápteros en una población local de Microtus m. mexicanus (Saussure) (INS.; SIPH.; MAMM.; CRICET). Tesis doctoral, Facultad de Ciencias UNAM: 124 + XIII.

Morrone, J. J. \& Gutiérrez, A. 2005. Do fleas (Insecta: Siphonaptera) parallel their mammal host diversification in the Mexican Transition Zone? Journal of Biogeography, 32, 1315-1325.

Olsen. O. W. 1962. Animal Parasites. Their Biology and Life Cycles. Burgess Publishing Company. Minneapolis, Minnesota. USA. 346 pp.

Pérez-Ortiz, T. 1976. Distribución de Siphonaptera en el derrame lávico del Chichinautzin, Morelos: Su interpretación ecológica y biogeográfica. Tesis Profesional, Facultad de Ciencias, UNAM, México, 156 pp.

Pfaffenberger, G. S., K. Kemether \& D. de Bruin. 1985. Helminths of sympatric populations of kangaroo rats (Dipodomys ordii) and grasshopper mice (Onychomys leucogaster) from the high plains of eastern New Mexico. Journal of Parasitology, 71: 592-595.

Ponce H. E. \& Llorente, J. 1996. Siphonaptera, pp. 553-565, In: Llorente, J., García A.N. \& González E.S. (Eds.) Biodiversidad, taxonomía y biogeografía de artrópodos de México: Hacia una síntesis de su conocimiento. Instituto de Biología-UNAM, Conabio y Facultad de Ciencias-UNAM, México,D.F.

Pulido-Flores, G. 2009. Identificación de algunos vertebrados nativos y sus helmintos parásitos, en la Reserva de la Biósfera Barranca de Metztitlán, Hidalgo, México. In Nova Ciencia, 1: 4-8.

Pulido-Flores, G., S. Moreno-Flores \& S. Monks. 2005. Helminths of Rodents (Rodentia: Muridae) from Metztitlán, San Cristóbal, and Rancho Santa Elena, Hidalgo, México. Comparative Parasitology, 72: 186-192.

Rankin, J. S. 1945. Ecology of the helminth parasites of the small mammals collected from Northrup Canyon, Upper Grand Coulee, Washington. Murrelet, 26: 11-14.

Ramírez., S. 1982. Distribución altitudinal de los ácaros mesostigmados y los sifonápteros de roedores en el campo experimental forestal “San Juan Tetla” Puebla, México. Tesis profesional, Fac. Ciencias, UNAM: 169 pp.

Read, C. P. 1956. Trichuris dipodomis, n. sp., from Ord's Kangaroo Rat. Proceedings of the Helminthological Society of Washington, 23: 76-81.

Salceda-Sánchez, B. 2004. Clave para la identificación de adultos de las especies de pulgas (Insecta: Siphonaptera) comunes y de mayor importancia médica en México. Folia Entomológica Mexicana, 43: 27-41.

Salceda-Sánchez, B. \& M. W. Hastriter. 2006. A list of the fleas (Siphonaptera) of Mexico with new host and distribution records. Zootaxa, 1296: 29-43.

Sikes, R. S., W. L. Gannon, \& the Animal Care and Use Committee of the American Society of Mammalogists. 2011. Guidelines of the American Society of Mammalogists for the use of wild mammals in research. Journal of Mammalogy, 92: 235-253. 
Smit, F. G. A. M. 1957. Handbook for the identification of British insects (Siphonaptera). Vol. 1, Part. 16. Royal Entomological Society of London, London, U. K. 94 pp.

Traub, R. 1950. Siphonaptera of Central America and Mexico. A morphological study of the aedeagus with descriptions of new genera and species. Fieldiana Zoology, 1: 1-127.

Traub, R. \& C. Hoff. 1951. Records and descriptions of fleas from New Mexico. American Museum of Novitates, 1530: 1-23.

Valdez, M. \& G. Ceballos. 1997. Conservation of endemic mammals of Mexico: The Perote ground squirrel (Spermophilus perotensis). Journal of Mammalogy, 78: 74-82.

Whitaker, J. O., Jr., W. J. Wren \& R. E. Lewis. 1993. Parasites, pp. 386-478. In: H. H. Genoways \& J. H. Brown (Eds.). Biology of the Heteromyidae. Special publication No. 10. The American Society of Mammalogists.

Wilson, D. E., \& D. A. Reeder. 2005. Mammal species of the world. A taxonomic and geographic reference. The Johns Hopkins University Press, Baltimore. 